\title{
Heparinized silk fibroin hydrogels loading FGF1 promote the wound healing in rats with full-thickness skin excision
}

\author{
Sirong He ${ }^{1 \dagger}$, Dan Shi ${ }^{2 \dagger}$, Zhigang Han ${ }^{3}$, Zhaoming Dong ${ }^{4,5}$, Yajun Xie $^{6}$, Fengmei Zhang ${ }^{3}$, WenXin Zeng ${ }^{3}$ \\ and Qiying $\mathrm{Yi}^{3^{*}}$ (D)
}

*Correspondence:
qyi@cqmu.edu.cn
${ }^{+}$Sirong He and Dan Shi are
the first co-authors
${ }^{3}$ Laboratory Animal
Center, Chongqing
Medical University,
Chongqing 400016, China
Full list of author information
is available at the end of the
article
article

\begin{abstract}
Background: Silk fibroin hydrogel, derived from Bombyx mori cocoons, has been shown to have potential effects on wound healing due to its excellent biocompatibility and less immunogenic and biodegradable properties. Many studies suggest silk fibroin as a promising material of wound dressing and it can support the adhesion and proliferation of a variety of human cells in vitro. However, lack of translational evidence has hampered its clinical applications for skin repair. Herein, a heparin-immobilized fibroin hydrogel was fabricated to deliver FGF1 (human acidic fibroblast growth factor 1) on top of wound in rats with full-thickness skin excision by performing comprehensive preclinical studies to fully evaluate its safety and effectiveness. The wound-healing efficiency of developed fibroin hydrogels was evaluated in full-thickness wound model of rats, compared with the chitosan used clinically.
\end{abstract}

Results: The water absorption, swelling ratio, accumulative FGF1 releasing rate and biodegradation ratio of fabricated hydrogels were measured. The regenerated fibroin hydrogels with good water uptake properties rapidly swelled to a 17.3-fold maximum swelling behavior over $12 \mathrm{~h}$ and a total amount of $40.48 \pm 1.28 \%$ hydrogels was lost within 15 days. Furthermore, accumulative releasing data suggested that heparinized hydrogels possessed effective release behavior of FGF1. Then full-thickness skin excision was created in rats and left untreated or covered with heparinized fibroin hydrogels-immobilized recombinant human FGF1. The histological evaluation using hematoxylin and eosin (HE) and Masson's trichrome (MT) staining was performed to observe the dermic formation and collagen deposition on the wound-healing site. To evaluate the wound-healing mechanisms induced by fibroin hydrogel treatment, wound-healing scratch and cell proliferation assay were performed. it was found that both fibroin hydrogels and FGF1 can facilitate the migration of fibroblast L929 cells proliferation and migration.

Conclusion: This study provides systematic preclinical evidence that the silk fibroin promotes wound healing as a wound-healing dressing, thereby establishing a foundation toward its further application for new treatment options of wound repair and regeneration.

Keywords: Silk fibroin, Hydrogel, FGF1, Wound healing 


\section{Background}

The normal wound-healing process is divided into several predictable phases: hemostasis, inflammation, proliferation and new tissue remodeling [1]. Silk fibroin from the cocoons of silkworm, composed of heavy and light chains linked by a disulfide bond, has been widely explored as wound dressing biomaterials for skin repair due to its hemostatic properties, low inflammatory potential, gaseous permeation, waterproofness and biodegradability of the material to escape disrupting the wound site [2-5]. It has been shown that silk fibroin can provide good structural support for cell attachment spreading, proliferation and migration of keratinocytes and fibroblasts [6, 7]. Several studies have reported that silk fibroin in different forms such as sponges, films and hydrocolloid dressings has been explored for its therapeutic effect in wound dressing to promote the healing process [8-10]. Some of them were widely used in growth factors, controlled release carrier of drugs and scaffolds of cell culture $[11,12]$. It was implicated that regenerated silk fibroin hydrogel, a state of sol-gel transition, induced the healing process of different wound healing by increasing the cells proliferation and migration of various cells type. Moreover, the incorporation of growth factors into silk fibroin hydrogel can accelerate the wound-healing process. Despite the positive results for various silk biomaterials in vivo and in vitro studies to look for adequate evidence that are implicated in the wound-healing effects in different types of cells during the deposition of collagen and wound construction, however, little work has been done using silk fibroin hydrogel to support its potential application as a wound dressing in the biomedical field [13-17].

In the present study, silk fibroin hydrogel was firstly prepared and the release rate of the FGF1 from fibroin hydrogels was evaluated by immunodetection over a period of 5 days. The fibroin hydrogel properties such as water absorption, swelling behavior and in vitro degradation rate were analyzed in neutral conditions (in PBS, pH 7.4) at different time points. Moreover, the external morphology and porosity of silk fibroin hydrogel was observed by scanning electron microscopy. Secondly, full-thickness excisional wounds in SD (Sprague-Dawley) rats treated with silk fibroin hydrogels with or without FGF1 were used to evaluate in vivo wound-healing efficacy in comparison to commercial wound dressing product (positive control). The HE staining results demonstrated that fibroin hydrogels with FGF1 accelerated the dermis formation and epidermal differentiation into hair follicles and sebaceous glands at days 7 and 14. Furthermore, collagen deposition within fibroin hydrogels loading FGF1 was more stout and wavy, attributed to small scar healing. It has been demonstrated that FGF1 contributes to multiple cellular targets in vitro or diverse therapeutically relevant biological activities in animal models of tissue repair such as wound healing $[18,19]$. Thirdly, proliferation and migration of the L929 cells seeded on the surface of fibroin hydrogel directly and cultured for $24 \mathrm{~h}$ in the presence or absence of FGF1 were measured by EdU (5-ethynyl-20-deoxyuridine) labeling and "scratch" assay wound model. These results suggested that fibroin hydrogel has tremendous potential for wound healing in the field of tissue regeneration engineering.

\section{Result}

Regenerated silk fibroin hydrogel preparation and properties characteristics

The fabricated fibroin hydrogels from the cocoons of silkworm were thin and transparent (Fig. 1a, b). The porosity and pore size of fibroin hydrogels measured using 
surface area and pore characterization system (ASAP 3020; Micromeritics Instrument Corporation Tristar) were $92.0627 \%$ and $8.9605 \mu \mathrm{m}$, respectively. Scanning electron microscope (SEM) was used to study the morphology of the silk fibroin hydrogels with lamellar, porous and interconnected microstructures (Fig. 1c, d), which may allow the cells to move within the scaffold in the process of wound healing and regeneration. The water absorption ratio of fibroin hydrogels was measured at different time points after soaking in PBS ( $\mathrm{pH}$ 7.4) solution. The ratio of water absorption was gradually increased to its peak by $8 \mathrm{~h}$ and decreased rapidly over the remaining $10 \mathrm{~h}$ (Fig. 1e). The fibroin hydrogels showed rapid swelling to a 17.9-fold maximum in neutral conditions ( $\mathrm{pH}$ 7.4) over $12 \mathrm{~h}$ (Fig. 1g). The release of FGF1 from the heparinized fibroin hydrogels soaking in the FGF1 solution (Fig. 1f) contained two stages: rapid release where approximately $43.7 \%$ of the total FGF1 was released in the first $12 \mathrm{~h}$, then slow release where a cumulative $70 \%$ of the total FGF1 in the hydrogel was released over the remaining days. The fibroin hydrogels were rapidly degraded, with $15.5 \pm 1.89 \%$ of the material lost within the first 2 days (in PBS, pH 7.4), then the degradation rate decreased and a total amount of $32.1 \pm 2.36 \%$ loss was found within 16 days (Fig. 1h).

\section{Expressions of cytokine and growth factor in full-thickness skin defects}

Growth factors played an important role in regulating the cellular migration and proliferation in the inflammatory phase of wound-healing stage. PDGF and TGF- $\beta$ were examined by using ELISA at three time points. The relative fold change means different concentrations of growth factors in four operation groups divided by that of normal skins cut from rats. The expression levels of PDGF in silk fibroin hydrogel treated with FGF1 were the highest on day 7, which were significantly higher than those of the chitosan, fibroin hydrogel and blank groups (Fig. 2e) but decreased rapidly on day 10 and 14, with no statistical significant difference among them. Moreover, there was no statistical significant difference for expression of TGF- $\beta 1$ from day 7 to 14 (Fig. 2f).

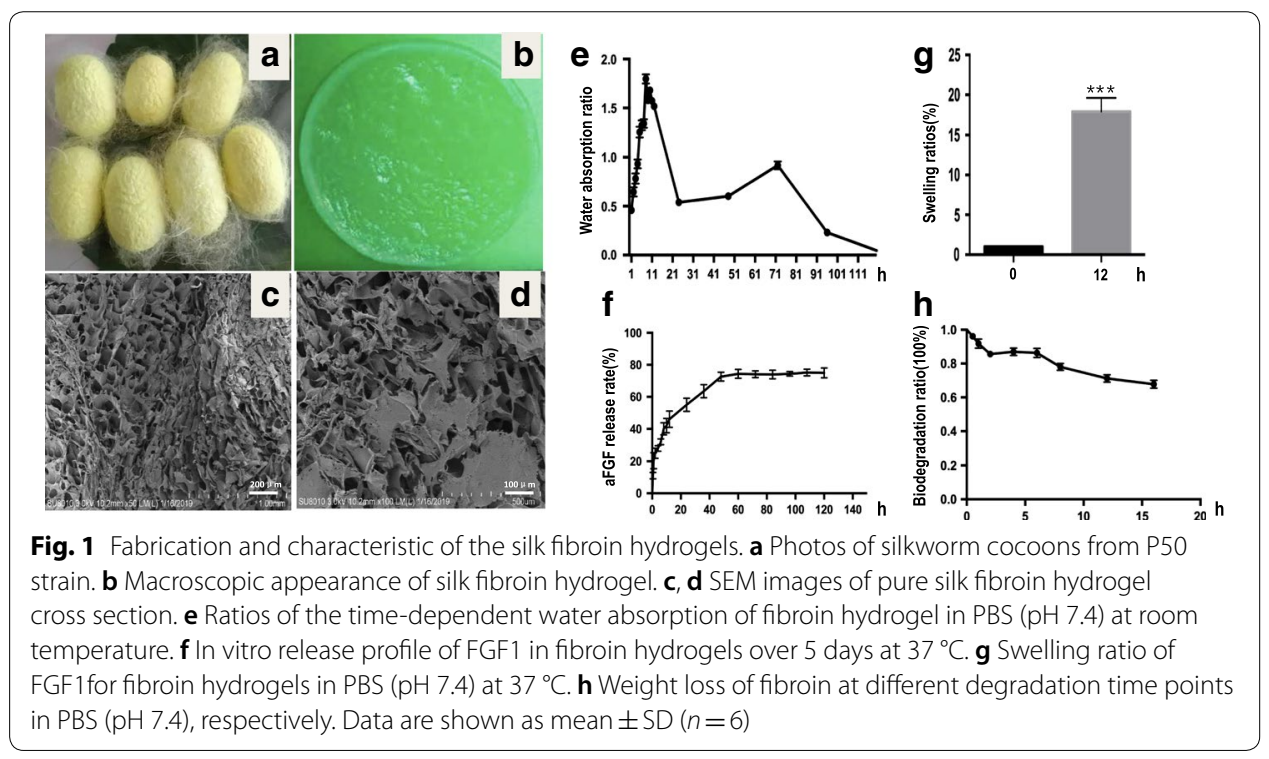




\section{Rat full-thickness skin defects model created and wound size measured}

The silk fibroin hydrogels prepared were applied on the full-thickness excisional wounds in rats (Fig. 2a). To visualize changes in wound size over time, photographs of the wound region were taken on days $7,10,14$ and 23 (Fig. 2b). The wound size reduction rate was calculated by comparing the wound size at each time point with the original wound size on day 0 . Initially, the wound size of chitosan- and silk fibroin-treated groups with or without FGF1 was significantly smaller than that of the blank control on day 7, but no significant difference between them was detected. Wound healing in chitosan- and fibroin hydrogel-treated groups were similar on the 10th day after operation. On day 14 postoperation, the wound size reduction rate of the heparinized silk fibroin with FGF1 reached $90.55 \%$, which was significantly higher than that of the untreated blank group (41\%, $P<0.001$ ) (Fig. 2c). After 23 days, all wounds of full-thickness skin excision were healed completely. In addition, the wounds treated with heparinized hydrogels with FGF1 had significantly reduced scar formation at the visual levels compared with the other groups (Fig. 2b). Our findings showed that the area of the wounds covered with silk biomaterials appeared smooth when compared with chitin wound dressing or blank control on day 23 (Fig. 2b). Taken together, silk fibroin hydrogels with FGF1 have excellent effect in promoting wound healing in rats.

\section{Histological observation of the wound-healing process}

To compare the wound-healing effect of silk fibroin hydrogels with chitin wound dressings, we further histologically evaluated the repaired tissue with HE staining at day 7 and 14. On day 7 postoperation, immature granulation tissue under epithelium

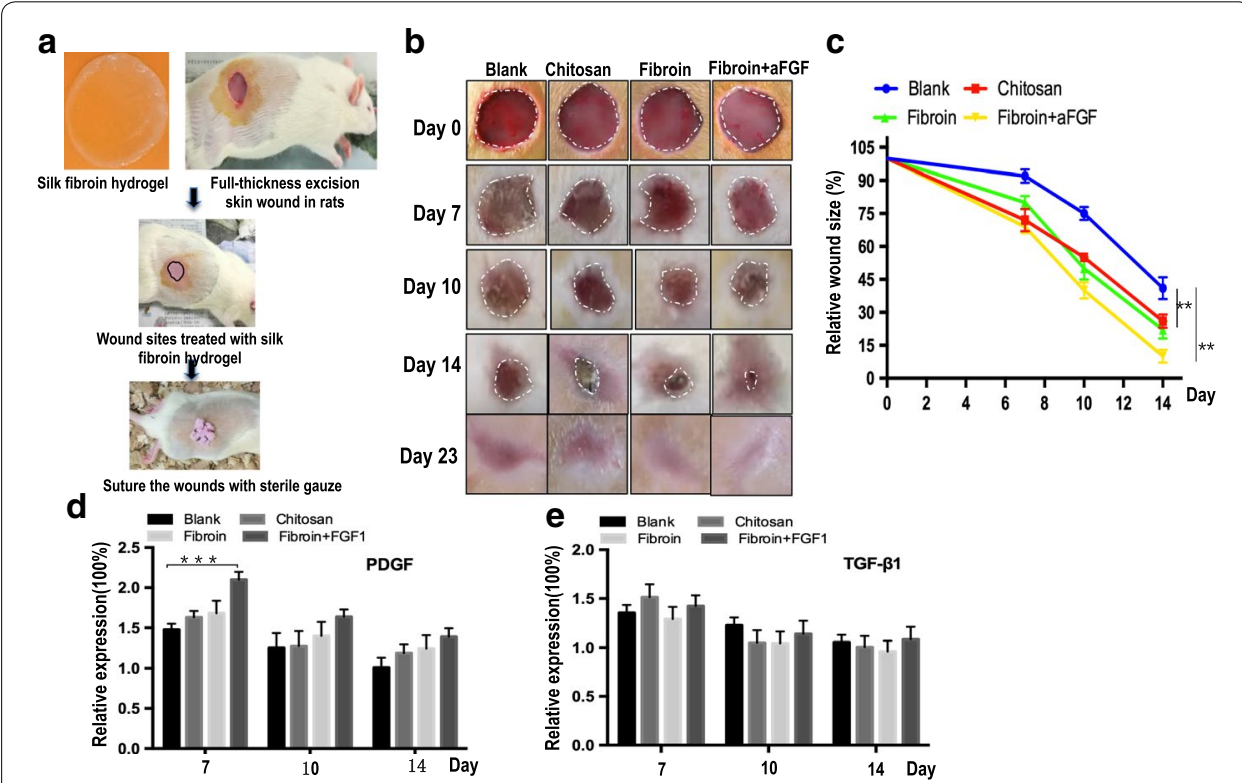

Fig. 2 a Schematic diagram of silk fibroin hydrogel application for wound healing. The wound was formed by punching a hole through the whole skin of rats. $\mathbf{b}$ Extent of wound healing on days 7,10 and 14. c Measure changes in the percentages of wound closure. $\mathbf{d}$, e The expression levels of PDGF and TGF- $\beta 1$ in full-thickness skin defects wound using ELISA at three time points. The relative fold change was ratios between different concentration of growth factors in four operation groups and normal skins cut from rats at different time points. Asterisks indicate statistical significance based on Student's $t$ tests $(* * P<0.001)$ 
was evident with loose collagen matrix present in the defect region of the blank control group (Fig. 3). In addition, the structure of the dermis in the blank group was not complete, with large blank areas on day 7 after operation. Compared with the empty group, silk fibroin hydrogel and chitosan dressings can better provide structural support for cell attachment, growth and migration on the wound sites; moreover, the structure of the new full-thickness skin growth healed by fibroin hydrogel and chitosan was intact, containing epidermis (E) and dermis (D) associated with hair follicles, adipose cells and muscle (yellow and black arrows in Fig. 3). After 14 days, well-defined dermal and epidermal layers with abundant collagen deposition and numerous capillaries and sebaceous glands were visible obviously in the wounds treated with fibroin hydrogel and chitosan, whereas in blank control wound, collagen fibers were less densely packed and contained a more porous structure (Fig. 3). Taken together, these data demonstrated that silk fibroin hydrogels with FGF1 could significantly improve wound-healing effect in rats.

\section{Effects of regenerated silk fibroin hydrogels on L929 cell growth and migration in the presence and absence of FGF1 in culture medium}

To understand the mechanism of the wound-healing effects of silk fibroin, firstly the EdU incorporation analysis was performed to visualize the proliferation in L929 cells. The results showed that only a small number of L929 cells from the blank group emitted a weak red fluorescence signal. There was similar luminous intensity between cells in medium with FGF1 or fibroin hydrogel group. However, more cells cultured in silk fibroin hydrogel in the presence or absence of FGF1 in culture medium exhibited intensive red fluorescence signal than the control group (Fig. 4a, b). These results suggested

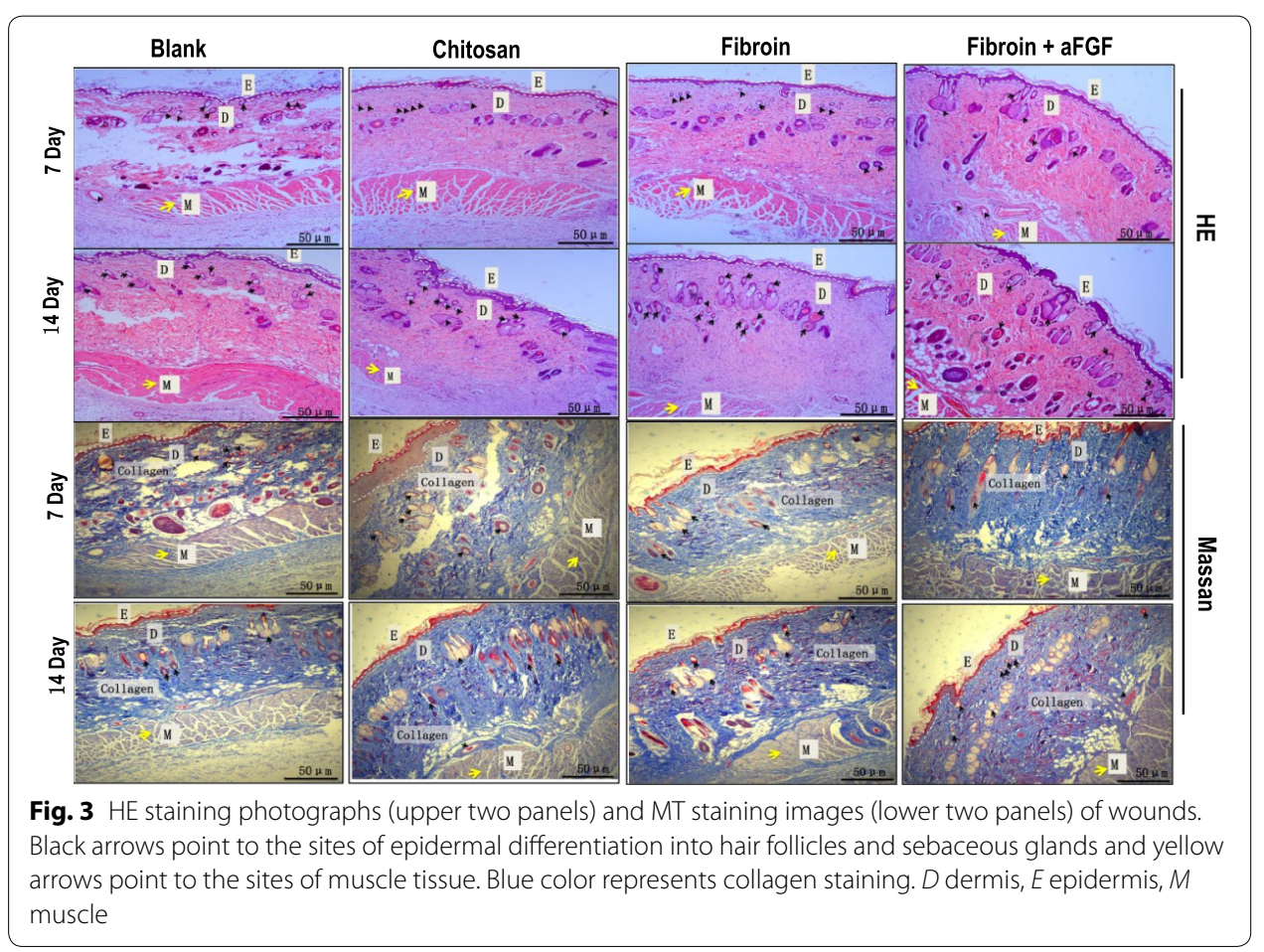


that silk fibroin hydrogel in the presence or absence of FGF1 promoted L929 cell proliferation. Furthermore, wound-healing assay showed more newly generated cells in fibroin hydrogel treated by FGF1 migrated into the scratch regions than the other treated groups (Fig. 4c, d).

\section{Discussion}

Silk fibroin, extensively explored as a promising biomaterial in different forms such as hydrogels, sponges, films and electrospun mats, had excellent biocompatibility, biodegradability and antimicrobial properties to promote wound healing and less scar formation [9, 23-26]. Moreover, many studies showed that silk fibroin produces less inflammation and low immunogenicity [27]. It has been a long time to apply silk fibroin for wound treatment [28]. In the present study, water absorption, swelling ratio and biodegradability of fibroin hydrogels were evaluated at different time points. It was reported that FGF1 release from fibroin hydrogel increased with decrease in fibroin concentration $[29,30]$. It is indicated that silk fibroin hydrogel produced a gradual increase and sustained the concentration of FGF1 over a long period, which was suitable for the application of drug delivery. Morphological analysis of the internal structure of fibroin hydrogels showed lamellar, porous and interconnected microstructure, which could provide convenient spaces for cell growth and transport of bioactive molecules. According

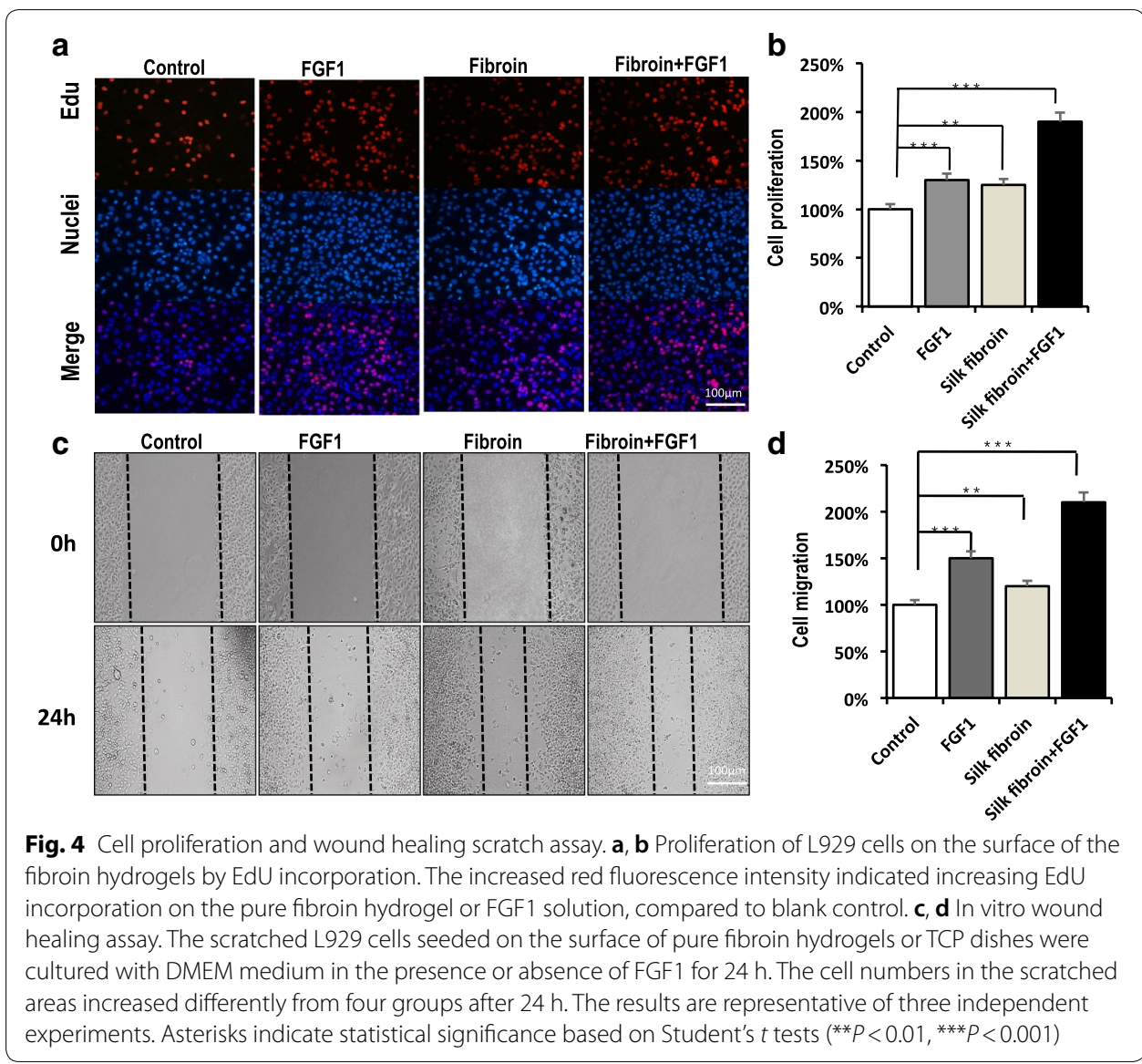


to the previous research, we selected a lower fibroin concentration to form hydrogel in this study. Prior to the clinical trial, the safety and effectiveness of the fibroin hydrogel should be firstly evaluated in animal model. Considering that human FGF1 has potential therapeutic applications for accelerating wound repair and clinical treatment of chronic non-healing dermal ulcers in diabetes [31-34]. In the present study, full-thickness cutaneous defect on the back of rats were firstly established. Fibroin hydrogels prepared with FGF1 were carried out to cover wound sites to release FGF1 slowly. In this study, heparinized hydrogels loading FGF1 is superior to improve overall wound healing and substantially decrease the time required to achieve total closure, as demonstrated by fast wound closure, re-epithelization and earlier formation of granulation tissue, which were remarkably faster than in the other groups. To better understand wound-healing mechanisms induced by fibroin hydrogel treatment, wound-healing scratch and cell proliferation assay were performed and showed that fibroin hydrogels or FGF1 can facilitate the migration of fibroblast L929 cells, which may provide evidence for cell proliferation and growth in wound sites.

The aim of this study was to investigate the possibility of using wound dressings made of silk fibroin hydrogels functionalized with FGF1 to help the closure of wounds by epidermidalization, dermis, angiogenesis and dense collagen deposition. What is more, silk fibroin has an easy availability from sericulture industry with low cost than the commercially available chitin dressings. All in all, if the aforementioned properties of silk fibroin hydrogel can be utilized and harnessed effectively in the future, there is no doubt that silk fibroin can be developed into new biomaterial and widely used in clinical treatment.

\section{Conclusion}

The aim of this study was to investigate the possibility of using silk fibroin as wound dressing functionalized with FGF1 to help the closure of wounds by increasing woundhealing rate, reepithelialization, dermis formation, collagen synthesis and epidermal differentiation into hair follicles and sebaceous glands compared to chitosan commercially available in market during the whole wound-healing period. The gross study on animal models indicates a visual pattern in which wounds treated with fibroin hydrogels show faster healing. Wound-healing scratch and cell proliferation assay showed that fibroin hydrogels with FGF1 facilitate the migration of fibroblast L929 cells, which is consistent with that obtained in the animal experiment.

Altogether, the results indicated that the functionalized silk fibroin can be developed into new biomaterial and widely used in clinical treatment.

\section{Methods}

\section{Preparation of silk fibroin hydrogels}

Fabrication of the silk fibroin hydrogel was carried out as in the previous published protocols [20]. Cocoons (supplied by Southwest University) were degummed in 0.05\% (W/V) $\mathrm{Na}_{2} \mathrm{CO}_{3}$ solution at $85{ }^{\circ} \mathrm{C}$ for $30 \mathrm{~min}$. The fibroin extract was then rinsed three times in Millipore water to extract the glue-like sericin proteins and wax. Subsequently, degummed silk fibroin was solubilized in $9.3 \mathrm{M} \mathrm{LiBr}$ (lithium bromide) solution for $4 \mathrm{~h}$ at $60{ }^{\circ} \mathrm{C}$, yielding a $20 \%$ solution $(\mathrm{w} / \mathrm{v})$. The mixing silk and $\mathrm{LiBr}$ solution obtained is a viscous and honey-like solution. This solution was then filtered through the dialysis 
membrane (MWCO 3500, Pierce) for 3 days to remove the salts and chemicals and then obtain about $8 \%(\mathrm{~W} / \mathrm{V})$ silk fibroin in aqueous solution, determined by weighing the remaining solid after drying. The silk fibroin solution with an initial concentration of $8 \mathrm{wt} \%$ was dialyzed against a $10-25 \mathrm{wt} \%$ PEG $(10,000 \mathrm{~g} / \mathrm{mol})$ solution at room temperature. After 9 h, $21 \mathrm{wt} \%$ silk fibroin solution was slowly collected by syringe to avoid excessive shearing and the concentration was determined. $2.3 \mathrm{wt} \%$ was prepared by diluting the $8 \mathrm{wt} \%$ solutions with distilled water. $5 \mathrm{ml}$ of fibroin solution was prepared in each conical tube and the sonication time was $30 \mathrm{~s}$ at the $20 \%$ amplitude setting. Then, on immediate transfer of the sonicated solution to a Petri dish and incubation of the plate at $37{ }^{\circ} \mathrm{C}$ for 3 days, circular-shaped gels sized $50 \mathrm{~mm}$ in diameter and $10 \mathrm{~mm}$ in thickness were formed. Hydrogel formation was observed when the sample presented an opaque white color and did not fall when the dish was inverted for $30 \mathrm{~s}$. The silk fibroin hydrogels were then subjected to freeze at $-20^{\circ} \mathrm{C}$ for $24 \mathrm{~h}$, and kept at $8{ }^{\circ} \mathrm{C}$ for $24 \mathrm{~h}$.

\section{Heparin immobilization and recombinant human FGF1 treatment of silk fibroin hydrogels}

The approach of heparin immobilization was based on previous studies [21, 22]. Heparin sodium salt (Sigma, H3149) was dissolved in a $10 \%$ sodium citrate aqueous buffer solution $(\mathrm{pH}=4.7)$ containing EDCI and refrigerated at $4{ }^{\circ} \mathrm{C}$ for $2 \mathrm{~h}$ to activate the heparin. The fibroin hydrogels prepared were then immersed in the heparin aqueous solution for $24 \mathrm{~h}$ at $4{ }^{\circ} \mathrm{C}$ to obtain heparin-immobilized hydrogel. After heparin was covalently immobilized on the surface of the hydrogels and washed with citric acid, $0.1 \%$ Triton $\mathrm{X}-100$ aqueous solutions, and subsequently rinsed with distilled water in an ultrasonic cleaner for $10 \mathrm{~min}$, it was finally dried in a vacuum dryer and kept in a refrigerator for use. $10 \mu \mathrm{g}$ of recombinant human FGF1 (from Pepro Tech 100-17A) was centrifuged for $1 \mathrm{~min}$ at $1000 \mathrm{rpm}$ prior to opening and dissolved in $5 \mathrm{mM}$ sodium phosphate (pH 8.0) buffer. All the heparinized and non-heparinized fibroin hydrogels were sterilized by a quick spray of $70 \%$ ethanol and a exposure under UV light for 15 min under sterile atmosphere. Lastly, heparinized hydrogels were soaked in recombinant human FGF1 solution $(1 \mu \mathrm{g} / \mathrm{ml})$ and allowed to swell for $2 \mathrm{~h}$ before using on the wound sites.

\section{Scanning electron microscopy (SEM)}

The fractured sections of silk fibroin hydrogel were cut using a razor blade after freezing in liquid nitrogen $\left(-196{ }^{\circ} \mathrm{C}\right)$. Fractured and surface sections of samples were vacuum coated with gold/palladium particles using the Polar on SC502 Sputter Coater. The morphology of fibroin hydrogel was examined with a field emission scanning electron microscope (FESEM) Zeiss EVO10 (Carl Zeiss AG, Germany) at $3 \mathrm{kV}$.

\section{Water uptake, swelling behavior and porosity measurement for fibroin hydrogels}

The water absorption rate of fibroin hydrogels was measured in different time points at room temperature in PBS (pH 7.4). The aqueous solution of the surface of each specimen was promptly removed. The weight change of the specimens was intermittently measured during each time point. The dynamic swelling ratios of the fibroin hydrogels were assayed according to the following equation: Swelling $(\%)=(\mathrm{Ws}-\mathrm{Wd}) / \mathrm{Wd} \times 100$. The dried and weighed fibroin hydrogels (Wd) were immersed in PBS, pH 7.4. At different time points, the swollen hydrogels were removed to determine the weight as Ws. The 
pore size and porosity of hydrogels were measured using the surface area and pore characterization system (ASAP 3020; Micromeritics Instrument CorporationTristar).

\section{In vitro analysis of FGF1 released from the fibroin hydrogels}

The silk fibroin hydrogels soaked in FGF1 solution were added into the six-well plates. Then, $1 \mathrm{ml}$ of PBS ( $\mathrm{pH}$ 7.4) was added to each well and kept at $37^{\circ} \mathrm{C}$. The supernatant from the wells was transferred to a tube and fresh PBS ( $\mathrm{pH}$ 7.4) of the same volume was added to the same well at different time points. FGF1 was collecting through the supernatant and then replaced by fresh solution. The amount of released FGF1 was analyzed by enzyme-linked immunosorbent assay kit (ELISA, Westang system, Shanghai, China). The FGF1 release system was carried out at $37^{\circ} \mathrm{C}$.

\section{Degradation properties of the fibroin hydrogel in vitro}

The hydrogel prepared was weighed (W1) in vitro before simulating the degradation testing. The samples were placed in $10 \mathrm{ml}$ PBS solution at $37{ }^{\circ} \mathrm{C}$ and weighed at different time points. W2 was the dry weight of composite hydrogel after drying for $12 \mathrm{~h}$. The fibroin hydrogel degradation rate was calculated by the equation of dry weight remaining ratio: $\mathrm{DWR}=\mathrm{W} 2 / \mathrm{W} 1 \times 100 \%$.

\section{Cell proliferation assay and in vitro wound-healing assay}

L929 cells were kindly provided by Stem Cell Bank, Chinese Academy of Sciences and cultured in DMEM medium with $10 \%$ fetal bovine serum (FBS) and 1\% penicillin/ streptomycin $(\mathrm{P} / \mathrm{S})$. The cell proliferation was measured using a Click-iTEdU Imaging Kit (Invitrogen) following the manufacturers' protocols. The labeled cells with EdU (5-ethynyl-20-deoxyuridine) incorporation were fixed with 3.7\% formaldehyde in PBS, and the EdU imaging was detected by fluorescence microscopy at the excitation/emission of 555/565 nm maxima. Analysis was conducted on fluorescence microscopes using single interference filters sets for blue (40,6-diamidino-2-phenylindole (DAPI)). For each interference filter, monochromatic images were acquired and merged using Cyto Vision (Leica Microsystems, Inc.). All cell images captured have the same exposure intensity and magnification. For determination of the percentages of EdU-positive cells, the red-fluorescent marked proliferated cells and (blue-fluorescent) DAPI marked the total cells, and the ratio between them represented the cell proliferation ability. All the slides were treated with the same threshold and the cell number was analyzed with the software Photoshop. Three independent experiments were performed for accuracy and the differences among the groups were compared. The ability of cell migration was determined using the wound-healing scratch assay. Cells were serum starved overnight at $37{ }^{\circ} \mathrm{C}$ in a $95 \%$ oxygenated tissue culture hood and scratched with a sterile clear tip. An amount of $200 \mathrm{ng}$ FGF1 in $1 \mathrm{ml}$ DMEM medium was used to culture the cells in silk fibroin hydrogels. Initial wounding and the migration of the cells in the scratched area after $24 \mathrm{~h}$ were photographically monitored using a microscope (Nikon, Tokyo, Japan) and calculated using Image J software. Measurements were performed in triplicate and repeated independently three times. 


\section{Implants in rats}

All SD rats were purchased from Hunan SLAC Laboratory Animal co., LTD (SCXK (Su) 2016-0002 and familiarized with their new environment for a week. All rats (250-350 g) used were male and fed under SPF (specific pathogen-free animal) facility with a 12:12 h light-dark cycle. All surgical procedures were conducted under animal care protocols approved by the Ethics Committee of Chongqing Medical University. Four rats were randomly assigned to each experimental group and distributed by four experimental groups with three time points. Sixty-four rats were randomly divided into four different groups [fibroin $(n=4)$, chitosan $(n=4)$, fibroin $+\operatorname{afgf}(n=4)$ and blank group $(n=4)$ ] at each time point: $7,10,14$ and 23 days. After anaesthetization (1.5-3 vol.\% Isoflurane), the dorsal surface hairs were shaved and then punched with a sterile $15 \mathrm{~mm}$ punch biopsy punch. Blank wound sites were covered with air-permeable Tegarderm ${ }^{\mathrm{TM}}$ (3 M, St. Paul, $\mathrm{MN}$ ) tape, while the other sites were covered with commercial chitin wound dressings or the silk fibroin hydrogels with or without FGF1. The commercial chitosan membranes were chose as the positive control (medical grade, Shanghai HaiChun Biotechnology Corporation). They were used to accelerate the wound healing and induce cell migration and proliferation in the market for many years due to many excellent properties such as low toxicity, good biocompatibility, antibacterial activity and so on. All rats were intraperitoneally injected with penicillin $(20,000 \mathrm{U} / \mathrm{kg} /$ days $)$ daily for 3 days. Rats were killed by $\mathrm{CO}_{2}$ exposure after each time point and samples were collected for histological examination. The images of the skin wounds in this study were captured using digital camera and imported onto Image J (v1.8.0) software. The extent of wound healing is expressed as the percentage of area remaining exposed. Wound size $(\%)=[R(7,10,14) / R(0)] \times 100$, where $R(0)$ and $R(7,10,14)$ represents the exposed wound area at postoperative days 0 and 7,10 , and 14 , respectively.

\section{Histological evaluation of the wounded skins healing in rats}

The silk fibroin hydrogels were cut into round shape and glued to the wound sites of rats. Rats were killed for histological analysis after healing for 7, 10 and 14 days, respectively. Specimens were harvested at these three time points and immediately fixed in $10 \%$ formalin solution at room temperature for at least $24 \mathrm{~h}$. Subsequently, paraffin-embedded tissue sections of $10 \mu \mathrm{m}$ thickness were obtained by using HE and MT staining for demonstration of skin architecture and collagen fibers by standard procedures.

\section{The expression analysis of PDGF and TGF $\beta$ released from the skin tissues of wound healing}

Rats were killed for assessment of PDGF and TGF $\beta$ at the indicated time points after healing by chitosan wound dressing and fibroin hydrogels with or without FGF1 for 7, 10 and 14 days. New skin tissues were disrupted using a Mini-BeadBeater-16 (Biospec) and homogenized with a tissue homogenizer following centrifugation $\left(750 g, 4^{\circ} \mathrm{C}\right)$ for $10 \mathrm{~min}$ after lysing in radio-immunoprecipitation (RIPA) buffer from Santa Cruz Biotechnology (Santa Cruz, CA, USA). Total protein concentration was determined by BCA Protein Assay Kit (Thermo Scientific). The samples were stored at $-80{ }^{\circ} \mathrm{C}$ and used for ELISA determination. 


\section{Statistical analysis}

GraphPad Prism v. 5.0 software (GraphPad Inc.; San Diego, CA, USA) was used for all the statistical analyses. Data represent mean $\pm \mathrm{SD}$. The statistical significance was

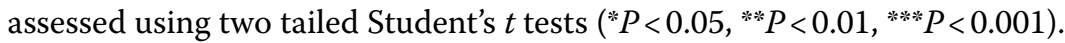

\section{Abbreviations}

FGF1: fibroblast growth factor-1; PDGF: platelet-derived growth factor; TGF- $\beta 1$ : transforming growth factor- $\beta 1$; ELISA: enzyme-linked immunosorbent assay; HE: hematoxylin and eosin; EDCl: 1-ethy-3-(-3dimethylamino-propyl) carbodiimide; MT: Masson's trichrome; SD: Sprague-Dawley; FBS: fetal bovine serum; SEM: scanning electron microscopy; EdU: 5-ethynyl-20-deoxyuridine; LiBr: lithium bromide; SPF: specific pathogen-free animal.

\section{Acknowledgements}

Not applicable.

\section{Authors' contributions}

QY gave the main idea, the whole design and completed the draft of the manuscript. ZD carried out the preparation of silk fibroin hydrogels and related property measurement. SH helped in assessment of the effectiveness and modification of the manuscript. SD participated in ELISA assay of the growth factors. All authors were in charge of establishing animal models with full-thickness cutaneous defect on the back of rats. YX was in charge of SEM, HE and MT staining. FZ and WZ helped to perform the cell proliferation assay and wound-healing scratch. All authors read and approved the final manuscript.

\section{Funding}

This work was supported by Chongqing Municipal Education Commission (No. KJ1702013); The Fundamental Research Funds for the Central Universities of China (No. XDJK2018C011); Science and Technology Planning Project in Dianjiang Country (No. djkjzdxm2018jsyfysfyy014); National Natural Science Foundation of China (No. 31702184).

\section{Availability of data and materials}

Data sharing not applicable to this article as no datasets were generated or analyzed during the current study.

\section{Ethics approval and consent to participate}

All the animals were anesthetized prior to extraction or implantation of wound dressings and were humanely euthanized after the procedures were completed. The animal experiments were approved by the Animal Center of ChongQing Medical University (ChongQing, China) and complied with the Instructive Notions with Respect to Caring for Laboratory Animals, 2006, published in by the Ministry of Science and Technology. This study was approved by the Ethics Committee of ChongQing Medical University with the Reference Number 2018020.

\section{Competing interests}

The authors declare that they have no competing interests.

\section{Author details}

${ }^{1}$ Department of Immunology, College of Basic Medicine, Chongqing Medical University, Chongqing 400016, China.

${ }^{2}$ Intervention Section, Chinese Medicine Hospital of Dianjiang County, Chongqing 408300, China. ${ }^{3}$ Laboratory Animal Center, Chongqing Medical University, Chongqing 400016, China. ${ }^{4}$ Biological Science Research Center, Southwest University, Chongqing 400716, China. ${ }^{5}$ Chongqing Engineering and Technology Research Center for Novel Silk Materials, Southwest University, Chongqing 400716, China. ${ }^{6}$ The M.O.E. Key Laboratory of Laboratory Medical Diagnostics, The College of Laboratory Medicine, Chongqing Medical University, Chongqing 400016, People's Republic of China.

Received: 5 April 2019 Accepted: 10 September 2019

Published online: 02 October 2019

\section{References}

1. Diegelmann RF, Evans MC. Wound healing: an overview of acute, fibrotic and delayed healing. Front Biosci. 2003;9(71):283-9.

2. Minoura N, Aiba SI, Higuchi M, et al. Attachment and growth of fibroblast cells on silk fibroin. Biochem Biophys Res Commun. 1995;208(2):516.

3. Santin M, Motta A, Freddi G, et al. In vitro evaluation of the inflammatory potential of silk fibroin. J Biomed Mater Res. 1999:46(3):382-9.

4. Gil ES, Panilaitis B, Bellas E, et al. Functionalized silk biomaterials for wound healing. Adv Healthcare Mater. 2013:2(1):206-17.

5. Susie S. Dressing selection in chronic wound management. J Am Podiatr Med Assoc. 2002;92(1):24-33.

6. Min BM, Lee G, Kim SH, et al. Electrospinning of silk fibroin nanofibers and its effect on the adhesion and spreading of normal human keratinocytes and fibroblasts in vitro. Biomaterials. 2004;25(7):1289-97.

7. Hersel U, Dahmen C, Kessler H. RGD modified polymers: biomaterials for stimulated cell adhesion and beyond. Biomaterials. 2003;24(24):4385-415.

8. Roh DH, Kang SY, Kim JY, et al. Wound healing effect of silk fibroin/alginate-blended sponge in full thickness skin defect of rat. J Mater Sci Mater Med. 2006;17(6):547-52. 
9. Fini M, Motta A, Torricelli P, et al. The healing of confined critical size cancellous defects in the presence of silk fibroin hydrogel. Biomaterials. 2005;26(17):3527-36.

10. Lee OJ, Kim JH, Moon BM, et al. Fabrication and characterization of hydrocolloid dressing with silk fibroin nanoparticles for wound healing. Tissue Eng Regener Med. 2016;13(3):218-26.

11. Liu Q, Liu H, Fan Y. Preparation of silk fibroin carriers for controlled release. Microsc Res Tech. 2015;80:312-20.

12. Wang $X$, Wenk E, Matsumoto A, et al. Silk microspheres for encapsulation and controlled release. J Control Release. 2007;117(3):360-70

13. Lawrence BD, Marchant JK, Pindrus M, et al. Silk film biomaterials for cornea tissue engineering. Biomaterials. 2009;30(7):1299-308.

14. Gil ES, Mandal BB, Park SH, et al. Helicoidal multi-lamellar features of RGD-functionalized silk biomaterials for corneal tissue engineering. Biomaterials. 2010:31(34):8953-63.

15. Wharram SE, Zhang X, Kaplan DL, McCarthy SP. Electrospun silk material systems for wound healing. Macromol Biosci. 2010;10(3):246-57.

16. Wittmer CR, Claudepierre T, Reber M, et al. Biomedical applications: multifunctionalized electrospun silk fibers promote axon regeneration in the central nervous system. Adv Funct Mater. 2011;21(22):4232-42.

17. Guo S, Pietro D. Factors affecting wound healing. J Dent Res. 2010;89(3):219-29.

18. Long Z, Qi H, Lu T, et al. TAT-mediated acidic fibroblast growth factor delivery to the dermis improves wound healing of deep skin tissue in rat. PLOS ONE. 2015;10(8):e0135291.

19. Huang L, Wang F, Wang Y, et al. Acidic fibroblast growth factor promotes endothelial progenitor cells function via Akt/FOXO3a pathway. PLOS ONE. 2015;10:e0129665.

20. Rockwood DN, Preda RC, Yucel T, Wang X, Lovett ML, Kaplan DL. Materials fabrication from Bombyx mori silk fibroin. Nat Protoc. 2011;6:1612-31.

21. Murugesan S, Xie J, Linhardt RJ. Immobilization of heparin: approaches and applications. Curr Top Med Chem. 2008;8(2):80-100

22. Kundu B, Rajkhowa R, Kundu SC, et al. Silk fibroin biomaterials for tissue regenerations. Adv Drug Deliv Rev. 2013;65(4):457-70.

23. Kimura T, Yamada H, Tsubouchi K, et al. Accelerating effects of silk fibroin on wound healing in hairless descendants of Mexican hairless dogs. J Appl Sci Res. 2007;3(11):1306-14.

24. Schneider A, Wang XY, Kaplan DL, et al. Biofunctionalized electrospun silk mats as a topical bioactive dressing for accelerated wound healing. Acta Biomater. 2009;5(7):2570-8.

25. Pandiarajan J, Cathrin BP, Pratheep T, et al. Defense role of the cocoon in the silkworm Bombyx mori L. Rapid Commun Mass Spectrometry Rcm. 2011;25(21):3303-6.

26. Guo X, Dong Z, Zhang Y, et al. Proteins in the Cocoon of silkworm inhibit the growth of Beauveria bassiana. PLoS ONE. 2016;11(3):e0151764.

27. Sugihara A, Sugiura K, Morita H, et al. Promotive effects of a silk film on epidermal recovery from full-thickness skin wounds. Proc Soc Exp Biol Med. 2000;225(1):58-64.

28. Altman GH, Diaz F, Jakuba C, et al. Silk-based biomaterials. Biomaterials. 2003:24:401-16.

29. Min HK, Park WH. Chemically cross-linked silk fibroin hydrogel with enhanced elastic properties, biodegradability, and biocompatibility. Int J Nanomed. 2016;11:2967-78.

30. Fang JY, Chen JP, Leu YL, et al. Characterization and evaluation of silk protein hydrogels for drug delivery. Chem Pharm Bull. 2006;54(2):156-62.

31. Wu J, Zhu J, He C, et al. comparative study of heparin-poloxamer hydrogel modified bFGF and aFGF for in vivo wound healing efficiency. ACS Appl Mater Interfaces. 2016. https://doi.org/10.1021/acsami.6b06047.

32. Maddaluno L, Urwyler C, Werner S. Fibroblast growth factors: key players in regeneration and tissue repair. Development. 2017;144(22):4047-60.

33. Xie L, Zhang M, Dong B, et al. Improved refractory wound healing with administration of acidic fibroblast growth factor in diabetic rats. Diabetes Res Clin Pract. 2011;93(3):403.

34. Liya H, Chen S. GW25-e0833 acidic fibroblast growth factor promotes the function of endothelial progenitor cells through Akt/FOXO3a signaling pathway. J Am Coll Cardiol. 2014;64(16):C64.

\section{Publisher's Note}

Springer Nature remains neutral with regard to jurisdictional claims in published maps and institutional affiliations.

Ready to submit your research? Choose BMC and benefit from:

- fast, convenient online submission

- thorough peer review by experienced researchers in your field

- rapid publication on acceptance

- support for research data, including large and complex data types

- gold Open Access which fosters wider collaboration and increased citations

- maximum visibility for your research: over $100 \mathrm{M}$ website views per year

At BMC, research is always in progress.

Learn more biomedcentral.com/submissions 